\title{
Machine for Producing Square Torsion Pulses of Microsecond Duration*
}

\author{
D. P. Pope, T. VReEland, JR., and D. S. Wood \\ W. M. Keck Laboratory of Engineering Materials, California Institute of Technology, Pasadena, California
}

(Received 14 April 1964; and in final form, 16 June 1964)

\begin{abstract}
A dynamic torsion testing machine has been built for the purpose of applying a known constant shear stress to a 12.7-mm-diam cylindrical test specimen for very short periods of time. The shear stress at the surface of the specimen rises from zero to any desired value in the range 0 to $3000 \mathrm{psi}$, within a period of 4 to $6 \mu \mathrm{sec}$. The time at constant stress varies linearly with axial position from the free end of the specimen ranging from 0 to about $500 \mu \mathrm{sec}$. The stress is removed within a period of 4 to $6 \mu \mathrm{sec}$ and for short specimens it remains essentially zero thereafter. This machine has been developed for the measurement of dislocation velocities up to $25 \mathrm{~m} / \mathrm{sec}$ in metal single crystals.
\end{abstract}

\section{INTRODUCTION}

$T$ HE apparatus described in this paper has been designed and built to study dislocation mobility in zinc and other metals. A brief description of this mobility experiment is given here to indicate the origin of the important parameters in the design of the machine. The method used to measure dislocation velocities is the following: Fresh dislocations are produced in a specimen by a very localized deformation. The specimen is then etched to reveal the initial dislocation configuration. A single stress pulse of known duration and amplitude is applied to the specimen. The specimen is etched again to determine the final dislocation configuration. Velocities are deduced from the dislocation displacements and the duration of the stress pulse. This procedure has been used by Johnston and Gilman ${ }^{1}$ and was refined by Stein and Low. ${ }^{2}$ Dislocation velocities of up to $25 \mathrm{~m} / \mathrm{sec}$ in metal single crystals are to be measured in this study. The specimens are 12.7 -mm-diam cylinders, although other sizes could be used if appropriate revisions were made on the machine. Dislocation etchants typically reveal only those dislocations in single crystals which thread the specimen surface within a few degrees of low index crystallographic planes. ${ }^{3}$ Thus, if the total angle in which dislocations can be revealed on a specimen surface is $\pm 5^{\circ}$ from a given direction, the maximum measurable dislocation displacement on a 12.7 -mm-diam specimen is $\left[\left(10^{\circ} / 180\right) \pi \times 6.35\right.$ $\mathrm{mm}] \approx 1.27 \mathrm{~mm}$. Thus the stress pulse must have a duration as small as the order of $1.27 \times 10^{-3} \mathrm{~m} / 25 \mathrm{~m} \cdot \mathrm{sec}^{-1}=50$ $\times 10^{-6} \mathrm{sec}$. However it is possible in some crystals to drive dislocations from one zone of dislocation etching to another and thereby greatly increase the measurable velocities. Since the stress pulse duration must be so short it is necessary that the application and release of the stress be accomplished in the order of $1 \mu \mathrm{sec}$. This is necessary so that the stress can reach maximum amplitude within the pre-

* This work was sponsored by the U.S. Atomic Energy Commission, Contract No. AT(04-3)-473.

${ }^{1}$ W. G. Johnston and J. J. Gilman, J. Appl. Phys. 30, 1929 (1959).

2 D. F. Stein and J. R. Low, J. Appl. Phys. 31, 362 (1960).

${ }^{3}$ R. C. Brandt, K. H. Adams, and T. Vreeland, Jr., J. Appl. Phys. 34,587 (1963).

scribed allowable time duration for measurements of high velocities. Such small rise, duration, and decay times of the stress can only be achieved by employing propagating stress waves in the material. These stress waves must be as simple as possible so that the stress-time history of the specimen is accurately known. Velocities can then be calculated by dividing the displacement by the pulse duration. A stress pulse of constant amplitude (square pulse) is optimum. This requirement eliminates longitudinal waves since they are dispersive in cylindrical rods. However, first mode torsional waves in isotropic rods, in the [0001] direction of cylindrical HCP crystals, and in

FIG. 1. Schematic of torsion impact machine.

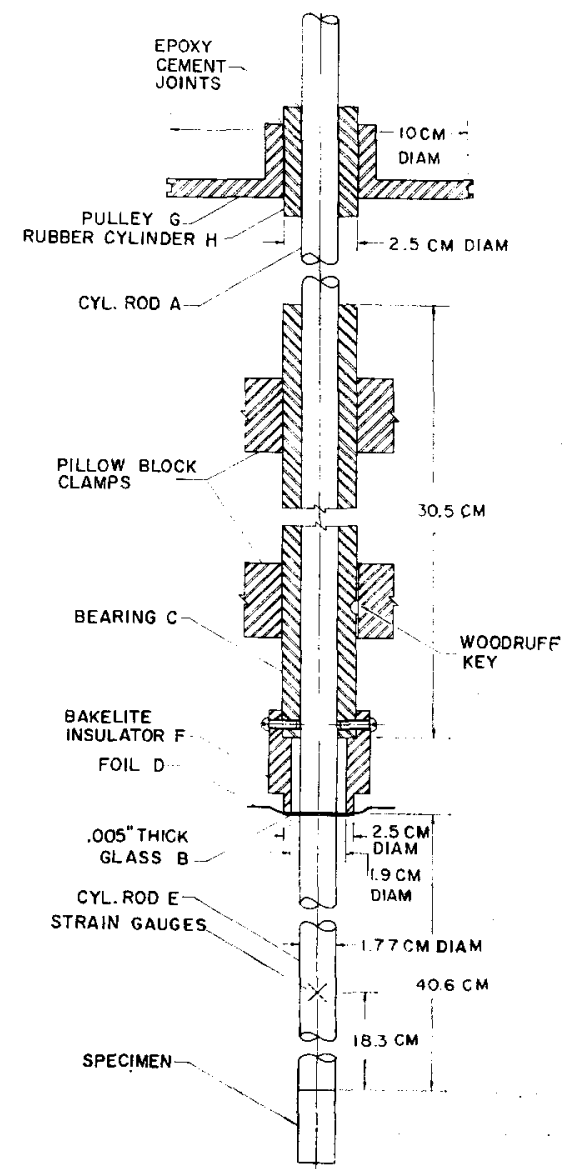




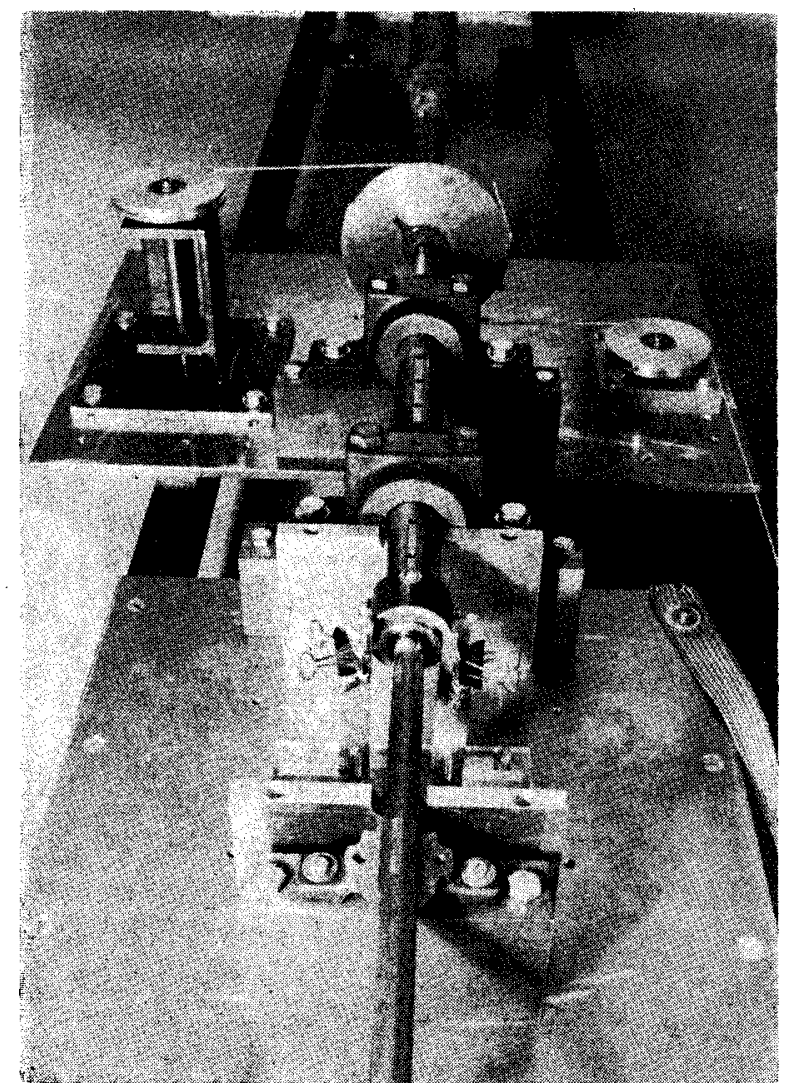

Fra. 2. Photograph of section of torsion impact machine.

the [100] direction of cylindrical cubic crystals are nondispersive. Torsion waves in cylindrical rods were chosen as the propagating stress waves.

It is also very important that only two stress wavefronts cross the specimen, one to load and the other to unload the specimen. Waves arriving several milliseconds after the initial loading wave must be carefully avoided to prevent additional stresses on the specimen.

Thus the torsion impact machine needed to perform these mobility experiments must have the following capabilities:

(1) It must produce a square nondispersive stress wave.

(2) The wave must have variable duration from 0 to several hundred microseconds.

(3) The pulse rise and decay time must be on the order of $1 \mu \mathrm{sec}$.

(4) It must apply one and only one stress pulse to a specimen. The machine designed herein quite effectively fulfills these requirements for short specimens.

\section{DESCRIPTION OF THE MACHINE}

The dynamic torsion testing machine that has been constructed employs propagating torsional waves in cylindrical rods. A schematic diagram of the apparatus is shown in Fig. 1. An initial static torque is applied to cylindrical rod A by means of the pulley G acting through the rubber sleeve $\mathrm{H}$. The thin glass disk $\mathrm{B}$ fastened to one end of the rod A by means of Eastman 910 cement transmits this torque to an insulator $\mathrm{F}$ which is attached to the fixed bearing C. A $0.006-\mathrm{mm}$-thick annular aluminum foil D is sandwiched between the glass disk and the insulator and all three of the elements are cemented together with Eastman 910 cement. One end of a second cylindrical rod $\mathbf{E}$ is cemented to the opposite side of the glass disk from rod A so that the center lines of the two rods coincide. Rod E does not carry static torque. A cylindrical test specimen is cemented to the free end of $\operatorname{rod} \mathbf{E}$, as shown. The rods $A$ and $\mathrm{E}$ are made of titanium for tests on zinc crystals having the [0001] crystal axis parallel to the cylindrical axis of the specimen. Since the torsional acoustical impedance of titanium is very close to that of [0001] oriented zinc crys-

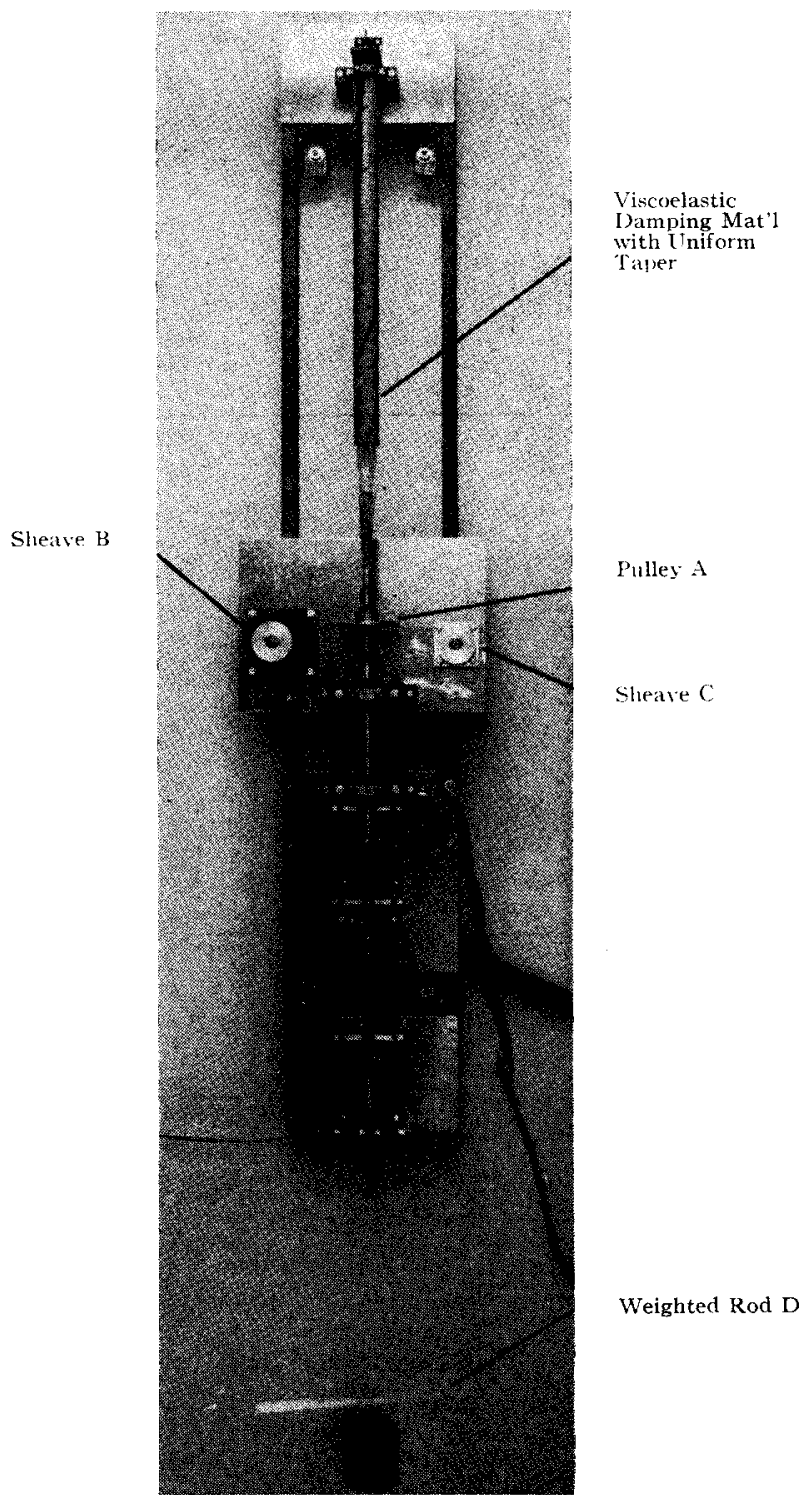

FiG. 3. Photograph of complete torsion impact machine. 
tals the torsion waves propagate unchanged through the interface between the specimen and rod $\mathbf{E}$.

Operation of the apparatus is initiated by a high voltage capacitor discharge through the foil $D$. This removes the bond between the glass disk $\mathrm{B}$ and the insulator $\mathrm{F}$ in 1 $\mu \mathrm{sec}$ or less, thus releasing the torque at that point. This sudden release of torque results in elastic torsional waves in rods $\mathrm{A}$ and $\mathrm{E}$. The wave in rod $\mathrm{E}$ propagates into the specimen and reflects from the free end of the specimen. The duration of stress at any point in the specimen is the time required for the wave to propagate from that point to the free end and return. The amplitude of the dynamic torque in rod $\mathrm{E}$ is one-half the initial static torque applied to $\operatorname{rod} \mathrm{A}$. Thus the magnitude of the stress in the test specimen can be adjusted to any value up to one-half the ultimate strength of the glass disk B or the cement. The amplitude and shape of the stress wave at the surface of rod $E$ is determined by means of silicon filament strain gauges together with a cathode ray oscilloscope and recording camera.

It is important that only one stress pulse be delivered to the specimen. This is accomplished, to a fair extent, by means of the following apparatus, shown in Figs. 1 and 3. In Fig. 1 the cylindrical sleeve of rubber $\mathrm{H}$, cemented with epoxy cement between the pulley $G$ and the rod $A$, has a very large torsional compliance. Thus a torsional wave is not significantly changed (reflected) as it passes under the pulley. The long portion of the rod A beyond the pulley $G$ is coated with a layer of polyurethane rubber. The thickness of the rubber varies uniformly from 0 at the pulley to $11.1 \mathrm{~mm}$ at the end of $\operatorname{rod} \mathrm{A}$. This viscoelastic damping material serves to absorb the waves in rod A. Thus, subsequent applications of the load to the specimen by reflecting waves from the free end of rod A are prevented.

Bending waves might be generated in rods $\mathrm{A}$ and $\mathrm{E}$ if the bond between glass disk $B$ and insulator $F$ is not released symmetrically around the circumference. However the foil $D$ is removed sufficiently rapidly and uniformly so that bending waves are not generated. The method of applying torque to pulley $\mathrm{F}$ can produce a small unbalanced

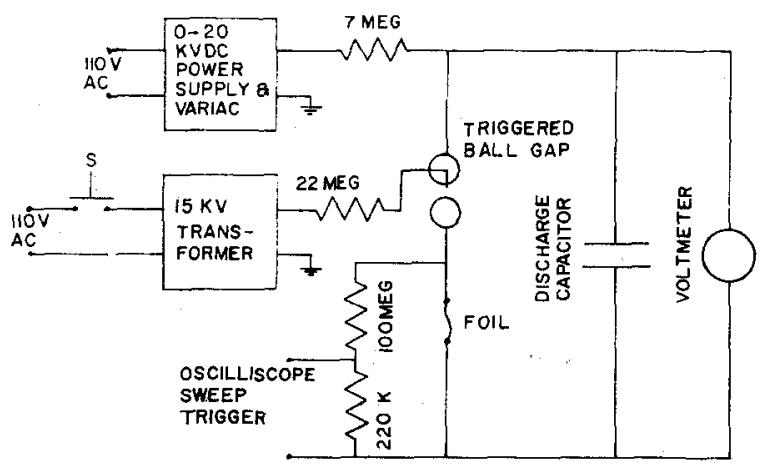

Fig. 4. Capacitor discharge circuit.

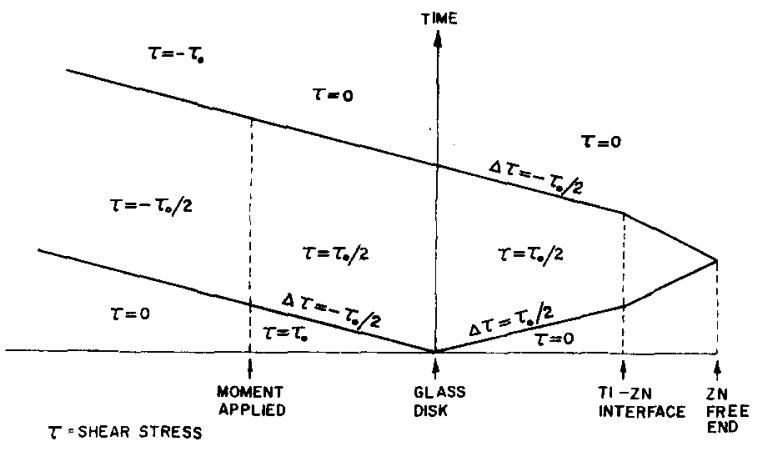

Fig. 5. Position-time diagram of stress waves.

force on $\operatorname{rod} A$. This could produce bending waves that would propagate down to the specimen after the foil is exploded. Bending waves are prevented by employing a diametrical clearance of less than $0.025 \mathrm{~mm}$ between rod $\mathrm{A}$ and bearing $\mathrm{C}$. This clearance is filled with SAE 30 oil which provides very effective damping for any flexural waves that might be generated in the system. The wave traverse time through the thickness of glass disk B is so small compared to the risetime of the torsional wave that reflections do not occur at the glass-metal interface.

Figures 2 and 3 are photographs of the apparatus. The method of torque application is shown in Fig. 3. A fine nylon cord is tied to opposite sides of pulley A. The cord passes over the low friction ball bearing sheaves B and C down to the weighted rod $D$. Since the pulley friction reduces the force in the cord by less than $1 \%$, the torque and hence the dynamic stress pulse amplitude are calculated directly from the weight on the rod. The strain gauges cemented to rod $\mathbf{E}$ in Fig. 1 provide an independent method for determining the stress applied to the specimen. They serve to show that the risetime and other aspects of the stress waves are behaving properly.

A $30^{\circ}$ taper joint just above the pulley $\mathrm{A}$ in Fig. 3 was included to make the system easier to disassemble. Another $30^{\circ}$ taper joint was included just below the strain gauges to facilitate specimen attachment. These taper joints are cemented together with Eastman 910 cement. After the stress has been applied to the specimen, it is necessary to etch it to determine where the dislocations have moved. Removing a zinc specimen from the bar without giving the specimen an undetermined amount of additional strain would be impossible. However, the Eastman 910 cement bond in the taper can be broken by rapid application of heat to the taper with the specimen in an isothermal bath to keep it at room temperature. The etching and photographing of the specimen can be done with the specimen still attached to the short rod. Tests have shown that these taper joints do not disturb the stress waves in the rods. Any disturbance of the wave would be seen as a kink near the center of the square stress pulse. This is not found.

The maximum stress amplitude that the system, shown 


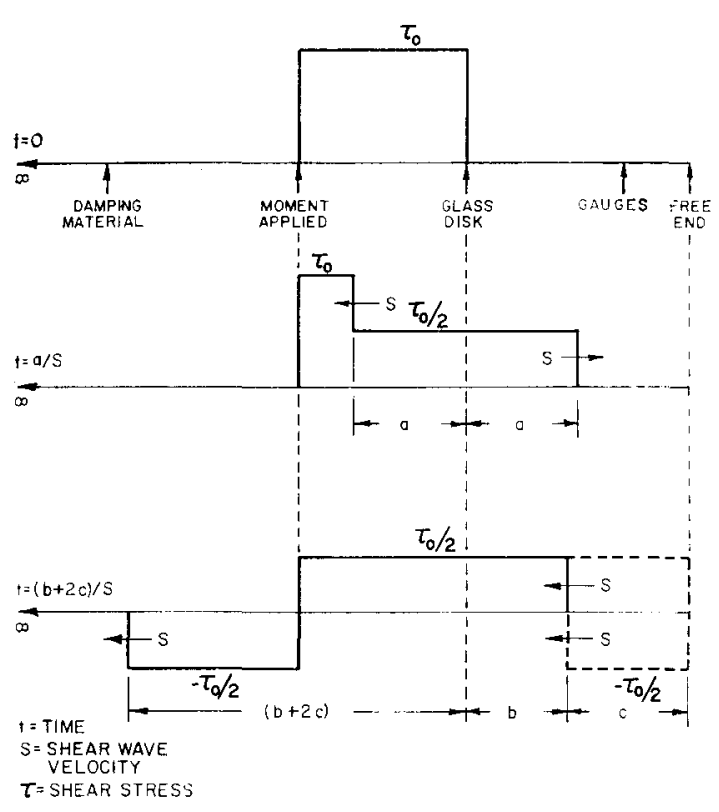

FIG. 6. Torsion waves in system at various times after intiation by foil removal.

in Fig. 1, can generate is determined by either the bond between $\operatorname{rod} \mathrm{A}$ and the glass disk B or by the ultimate strength of the glass disk. A bond strength of up to 6000 psi can be attained with epoxy cement rather than Eastman 910. A 3000-psi stress wave will then be generated in rod E. A metal disk could be used instead of the glass if

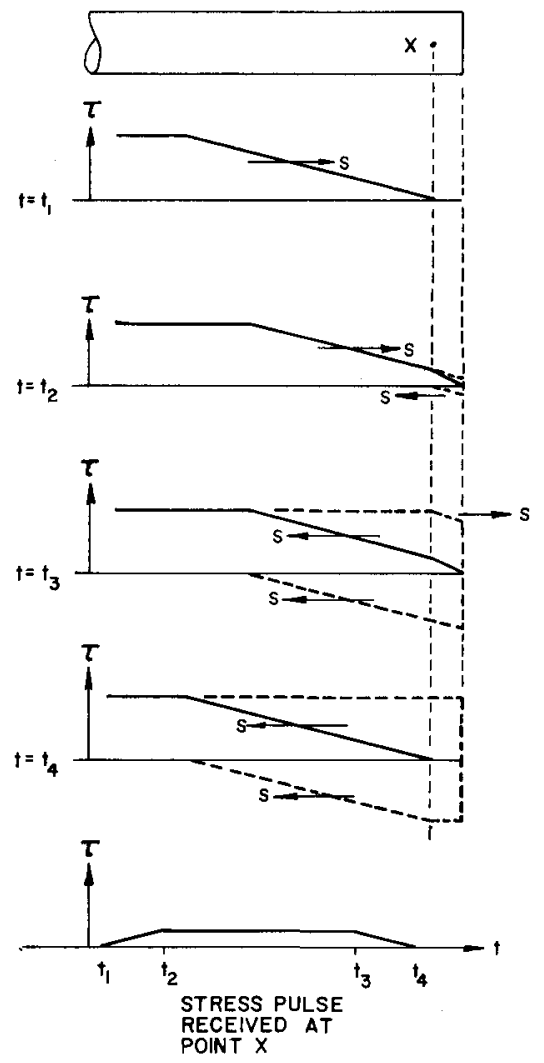

an insulating material were placed between the foil and the disk. Brazing the metal disk to the titanium would raise the upper stress limit of the system.

Figure 4 shows a partial schematic diagram of the capacitor discharge circuit. The power supply charges the $1-\mu \mathrm{F}$ capacitor to $10 \mathrm{kV}$ through the load resistor. This voltage is set by adjusting the Variac. When the capacitor is charged to $10 \mathrm{kV}$, the switch $\mathrm{S}$ is closed momentarily, sending a $15-\mathrm{kV}$ trigger pulse from the transformer into the ball gap. Except for this trigger pulse, the operation of this ball gap is the same as that of Cullington, Chace, and Morgan. ${ }^{4}$ This pulse causes the air gap to break down and the capacitor discharges to ground through the foil. The oscilloscope sweep is triggered by a $20-\mathrm{V}$ trigger pulse supplied by the voltage divider.

High sensitivity silicon crystal strain gauges $(1.27 \mathrm{~mm}$ long, $1500 \Omega$ nominal, gauge factor $=140$ ) obtained from Microsystems Inc., Pasadena, California, are mounted on the torsion rod to determine both torsional and flexural wave amplitudes. A torsion wave front traverses a strain gauge in approximately $0.3 \mu \mathrm{sec}$, so that the gauge essen-

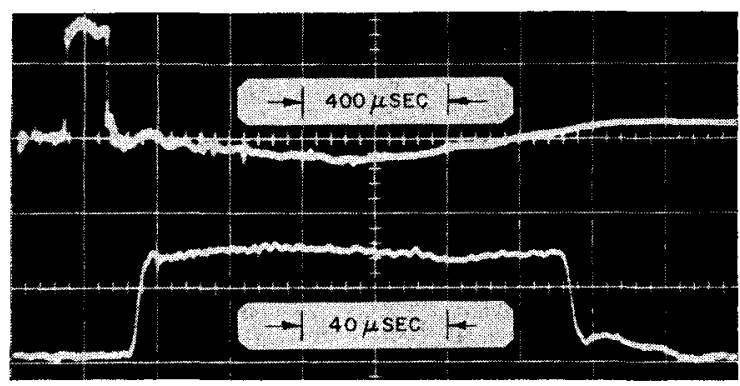

FIG. 8. Strain gauge signal from a 1060-psi torsional pulse.

tially indicates the strain at a point as a torsion wave with a $5-\mu \mathrm{sec}$ risetime passes the gauge. The strain gauges are connected in voltage divider circuits. It was found that stresses due to flexure are negligible.

The discharge of the capacitor through the spark gap and the exploding foil produce large amounts of electromagnetic radiation which can induce sufficient voltages in the strain gauge circuit leads to obliterate the pulse from the strain gauges. This pulse has an amplitude on the order of $20 \mathrm{mV}$. The foil is exploded at $10 \mathrm{kV}$. It is, therefore, necessary to carefully shield the strain gauge leads, to employ separate grounds for the strain gauge circuit and the capacitor discharge circuit, and to pass the strain gauge signals through low pass RC filters at the oscilloscope inputs. These filters have a $500-\mathrm{kc}$ corner frequency. To determine if the filters have any adverse effect on the strain gauge signals, the square-wave calibrate output on the Tektronix type 555 oscilloscope was viewed on the oscil-

${ }^{4}$ E. H. Cullingotn, W. G. Chace, and R. L. Morgan, ASTIA Rept. No. AD 84016 (September 1955). 
loscope screen and no difference was seen between the filtered and unfiltered waves. This square wave has a risetime about the same as the signal from the strain gauges.

\section{ANALYSIS OF WAVES AND MACHINE PERFORMANCE}

\section{A. Theoretical}

A theoretical position-time diagram for torsional waves in this system is shown in Fig. 5. Such a graphical analysis is analogous to that for a nondispersive longitudinal wave in a cylindrical rod. ${ }^{5}$ Note that the rubber-coated rod is assumed to be semi-infinite. This shows, as stated earlier, that the wave passes the point of static torque application without reflection.

Figure 6 shows waves in the system at various instants after the foil explodes. Figure 7 shows the stress as a function of time at a point near the end of the rod when a slowly rising stress wave occurs in the rod. In this case the stress pulse received at point $x$ is of much lower amplitude than the incident stress wave. This indicates that it is not necessary for there to be zero reflection from the rubber

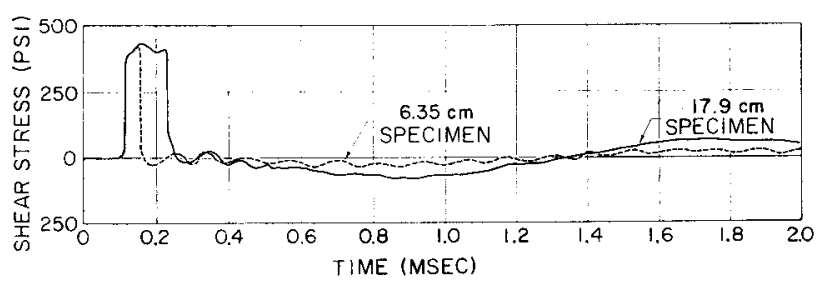

FIg. 9. Stress-time diagram at Ti-Zn interface for two different specimen lengths.

coated portion of the rod A, but only that the reflection be spread out with a slow risetime.

\section{B. Experiment}

Figure 8 is a photograph of two oscilloscope traces of the strain gauge signal from a 1060-psi amplitude pulse obtained from this system with no specimen attached to the rod. These traces were made from the same pulse using a Tektronix type 555 dual-beam oscilloscope with different time scales on the two traces. The upper trace is the stress during a 2-msec time period. The lower trace is the initial pulse expanded which shows that the pulse rise and decay times are about 4 to $6 \mu \mathrm{sec}$. Figure 9 shows the upper trace from Fig. 8 and the corresponding calculated stress that is applied to a point $6.35 \mathrm{~cm}$ from the free end of the rod. These are essentially the same stresses that would be applied to a $17.9-\mathrm{cm}$ - and a $6.35-\mathrm{cm}-\mathrm{long}$ specimen, respectively. These curves show that the polyurethane damp-

\footnotetext{
${ }^{5}$ K. J. DeJuhasz, Trans. ASME 64, A-122 (1942).
}

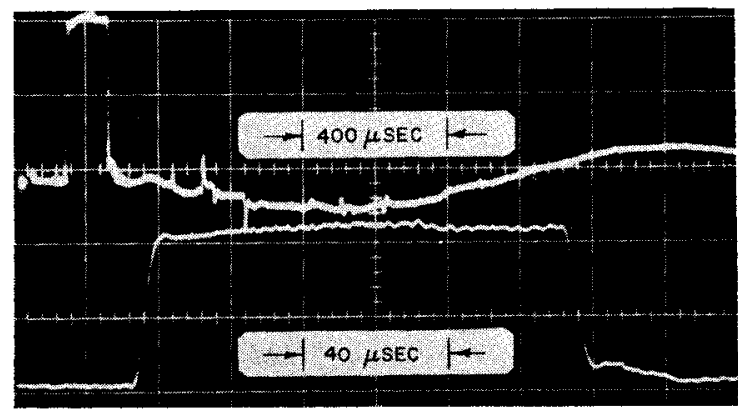

FIG. 10. Strain gauge signal from a 423-psi torsional pulse.

ing material does not immediately absorb all the torsional energy passing through it, but spreads it out in time. This is true for the following reason. As shown in Fig. 5, there would be only two wavefronts crossing the strain gauges (one to load, the other to unload) if the damping material did absorb all the torsional wave passing under it. These two fronts would yield a square pulse at the gauges with zero stress thereafter. But as shown in Fig. 8, there is a long period wave starting at about $400 \mu \mathrm{sec}$, the time corresponding to a reflection from the damping material. Note in Fig. 9 that the amplitude of this "noise" that actually enters a 6.35-cm-long specimen is far smaller than that which enters a 17.8-cm-long specimen. Thus for short specimens approximately $2.5 \mathrm{~cm}$ long this system is adequate, but in the future if specimens approximately $18 \mathrm{~cm}$ long are used, it will be necessary to modify the damping material to spread out the reflected wave even more.

Figure 10 shows a smaller amplitude pulse (423 psi). It appears that the stress could be further reduced without an adverse effect on the risetime.

Figure 11 shows the first part of a 423-psi pulse with an expanded time scale. The risetime is about $5 \mu \mathrm{sec}$.

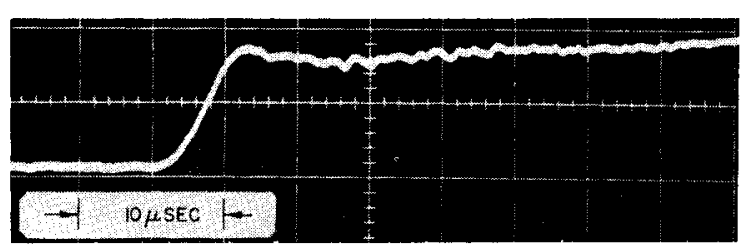

Fig. 11. Risetime of a 423-psi torsional pulse.

\section{ACKNOWLEDGMENTS}

The authors wish to thank R. C. Blish of California Institute of Technology for valuable help in the laboratory, G. J. Woffinden and R. R. Randall of Aerojet General Corporation, Downey, California, for assistance in designing and constructing the capacitor discharge circuit, and Dr. Donald S. Clark of California Institute of Technology for constructive criticisms of the manuscript. 